\title{
Impaired mitochondrial response to simulated ischemic injury as a predictor of the development of atrial fibrillation after cardiac surgery: In vitro study in human myocardium
}

\author{
Niv Ad, MD \\ Aviva Schneider, MSc ${ }^{\text {b }}$ \\ Igor Khaliulin, $\mathrm{PhD}^{\mathrm{b}}$ \\ Joseph B. Borman, $M D^{b}$ \\ Herzl Schwalb, PhD ${ }^{b}$
}

From The Department of Cardiothoracic Surgery ${ }^{\mathrm{a}}$ and The Joseph Lunenfeld Cardiac Surgery Research Center, ${ }^{\mathrm{b}}$ Hadassah University Hospital, Jerusalem, Israel.

Received for publication Sept 5, 2003; revisions requested March 15, 2004; accepted for publication March 25, 2004

Address for reprints: Niv Ad, MD, Cardiothoracic Surgery Department, Hadassah University Hospital, Jerusalem 91120, Israel (E-mail: nivadmd@hotmail.com).

J Thorac Cardiovasc Surg 2005;129:41-5

$0022-5223 / \$ 30.00$

Copyright $\odot 2005$ by The American Association for Thoracic Surgery

doi:10.1016/j.jtcvs.2004.03.058
Objective: Atrial fibrillation occurs in $20 \%$ to $40 \%$ of patients after cardiac surgery, but its pathophysiology remains unclear. Recent studies demonstrated preexisting histologic markers that portend the development of postoperative atrial fibrillation. In this prospective study, we focused on mitochondrial dysfunction in response to ischemic stress as a potential predictor for postoperative atrial fibrillation.

Methods: Slices of right atrial trabeculae from 50 patients undergoing elective cardiac surgery were surperfused with oxygenated glucose-containing phosphatebuffered saline solution. After 30 minutes of stabilization, the sections were exposed to 90 minutes of simulated ischemia (nitrogenated phosphate-buffered saline solution without glucose) followed by 90 minutes of reoxygenation (reintroduction of the oxygenated solution). Mitochondrial viability and response were measured by staining with 3-[4.5 dimethylthiazol 2-yl]-2,5-diphenyltetrazolium bromide. The magnitudes of mitochondrial recovery after simulated ischemia and 28 possible risk factors for postoperative atrial fibrillation were entered into univariate and multivariate models.

Results: There were no deaths in this group of patients. Nineteen patients (38\%) had postoperative atrial fibrillation. Interestingly, no difference in baseline (before simulated ischemia) mitochondrial function was documented between patients who had postoperative atrial fibrillation and those who did not. An independent predictor for postoperative atrial fibrillation was the degree of mitochondrial dysfunction in response to simulated ischemia, as measured by the intensity of the staining.

Conclusion: This study has identified for the first time an association between mitochondrial dysfunction in response to ischemia and postoperative atrial fibrillation. This finding improves our understanding of the pathophysiology of postoperative atrial fibrillation and may eventually lead us to identify candidates for selective preoperative or early postoperative prophylactic treatment.

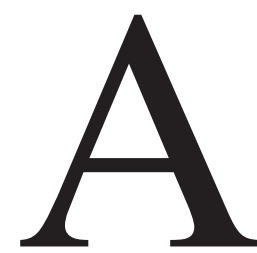

trial fibrillation is a common complication in cardiac surgery, occurring within the first week after operation in $20 \%$ to $40 \%$ of the patients undergoing cardiac surgery, and its incidence may be increasing. ${ }^{1}$ Although often regarded as a temporary, benign problem, this complication has significant adverse effects: it increases the risk of cerebrovascular accident and extends intensive care unit and hospital stays. ${ }^{2,3}$ 
TABLE 1. Characteristics of the study group $(n=50)$

\begin{tabular}{lc}
\hline Characteristic & Value \\
\hline Age (y, mean \pm SD) & $59.3 \pm 10.6$ \\
Male/female ratio & $2: 1$ \\
Coronary artery bypass grafting (No.) & 31 \\
Aortic valve replacement (No.) & 10 \\
Aortic valve replacement plus coronary artery bypass & 2 \\
$\quad$ grafting (No.) & 5 \\
Mitral valve surgery (No.) & 2 \\
Mitral valve surgery plus coronary artery bypass & \\
$\quad$ grafting (No.) & \\
\hline
\end{tabular}

The mechanism for the development of postoperative atrial fibrillation (POAF) is unclear. It is known that POAF involves reentry, resulting from disparate refractoriness between adjacent atrial areas, ${ }^{4}$ but the reasons for these dissimilarities are unknown. Risk factors associated with the development of POAF include advanced age, prolonged preoperative atrial conduction duration, chronic obstructive pulmonary disease, excess circulating catecholamines, electrolyte imbalance, atrial myocardial ischemia, sudden $\beta$-blocker withdrawal, prolonged aortic crossclamping, and right atrial manipulation. ${ }^{1-7}$ One of the confusing aspects of POAF is the fact that it does not develop in roughly $60 \%$ of patients having cardiac surgery, despite the presence of one or more of these risk factors in nearly all of them, suggesting an inherent preoperative risk of postoperative development in a subgroup of patients. This may be specifically suggested by the finding that prolonged preoperative atrial conduction duration is associated with POAF. ${ }^{6}$ It has previously been shown that high lipofuscin content and atrial myolysis found in atria before cardiopulmonary bypass (CPB) may also be associated with vulnerability to POAF. ${ }^{8,9}$ Taken together, these factors indicate that the preoperative status of a patient's atria is a major determinant of the risk of development of POAF, in addition to the stress and ischemia induced by the surgical procedure itself.

The association between advanced age and risk of atrial fibrillation after cardiac surgery is striking but specifically unclear. It has been attributed to age-related atrial changes such as atrophy and fibrosis, ${ }^{1,10}$ but this association has never been directly examined.

Susceptibility to POAF may depend on the preoperative metabolic status of the atria, and this may be reflected by the mitochondrial response to postischemic reperfusion injury. Directly or indirectly, mitochondria are important targets for virtually all types of injurious stimuli, including hypoxia and toxins. The damage is commonly expressed as the formation of high-conductance channels, the so-called mitochondrial permeability transition. This, together with findings of an association between deletion of mitochondrial DNA aging and atrial fibrillation, has led to the assumption that impaired mitochondrial function in atrial cardiomyocytes may be related to atrial fibrillation after cardiac surgery. ${ }^{11,12}$ This study was designed to examine mitochondrial function in response to simulated ischemia-reperfusion (I/R) stress as a potential predictor for the development of atrial fibrillation after surgery.

\section{Methods}

\section{Tissue Preparation}

The investigation conformed to the principles outlined in the Helsinki Declaration. Approval to conduct the study was obtained from the institutional ethics committee on human research (January 2002).

Right atrial appendages were obtained from 50 patients undergoing elective cardiac surgery with $\mathrm{CPB}$, but before $\mathrm{CPB}$ was initiated (Table 1). The protocol in use for simulated I/R of isolated human atrial sections was adopted from studies by Ghosh and colleagues. ${ }^{13}$ The specimens were placed in test tubes containing oxygenated, heparinized samples of patients' blood and immediately transferred to the laboratory. The specimens were rinsed with glucose-containing phosphate-buffered saline solution (G-PBS), and trabeculae were gently separated longitudinally.

Incubation medium. G-PBS ( $\mathrm{pH} 7.45$ ) was prepared daily in distilled water and contained the following: 136.9-mmol/L sodium chloride, 2.68-mmol/L potassium chloride, 8.10-mmol/L dibasic sodium phosphate, $1.53-\mathrm{mmol} / \mathrm{L}$ monobasic potassium phosphate, 0.5- $\mathrm{mmol} / \mathrm{L}$ magnesium chloride hexahydrate, $0.9-\mathrm{mmol} / \mathrm{L}$ calcium chloride dihydrate, and $5.55-\mathrm{mmol} / \mathrm{L}$ glucose.

Staining solution. The 3-[4,5-dimethythiazol-2-yl]-2,5-diphenyltetrazolium bromide (MTT) and diazoxide were obtained from Sigma Chemical Co. The MTT staining solution was prepared by dissolving $0.5 \mathrm{mg}$ MTT in $1 \mathrm{~mL}$ G-PBS.

\section{Simulated I/R}

Initially, atrial sections (5-10 mg each) were placed in containers with $20 \mathrm{~mL}$ G-PBS and incubated for 30 minutes for equilibration at $37^{\circ} \mathrm{C}$ in a cell culture incubator. After aerobic equilibration, the study sections were rinsed in phosphate buffered saline solution without glucose (PBS) and subjected to I/R, whereas the aerobic control sections remained in the incubator in oxygenated G-PBS for the entire experiment. Ischemia $\left(37^{\circ} \mathrm{C}\right)$ was simulated by placing the sections ( 2 sections per $35-\mathrm{mm}$ culture plate) in a sealed Plexiglas (Elf Atochem North America, Inc, Philadelphia, Pa) chamber (Figure 1). ${ }^{14}$ The chamber was vigorously flushed with $100 \%$ nitrogen for 5 minutes to replace the oxygen with nitrogen. En route to the chamber, the gas passed two traps: trap 1 contained $1 \%$ sodium sulfite and trap 2 contained water. A 2-mL syringe, flushed with nitrogen, was filled with ischemic PBS (without glucose, bubbled for 1 hour with nitrogen). Each well was quickly filled with $0.4 \mathrm{~mL}$ ischemic PBS (minimal volume to cover the slices) and corked. The flow of nitrogen was attenuated to approximately $1 \mathrm{bubble} / \mathrm{s}$ at the exit trap, and the ischemia was maintained for 90 minutes. At the end of 90 minutes of simulated ischemia, the slices were subjected to 90 minutes of reoxygenation in oxygenated G-PBS at $37^{\circ} \mathrm{C}$ in a $100 \%$ oxygenated environment. 


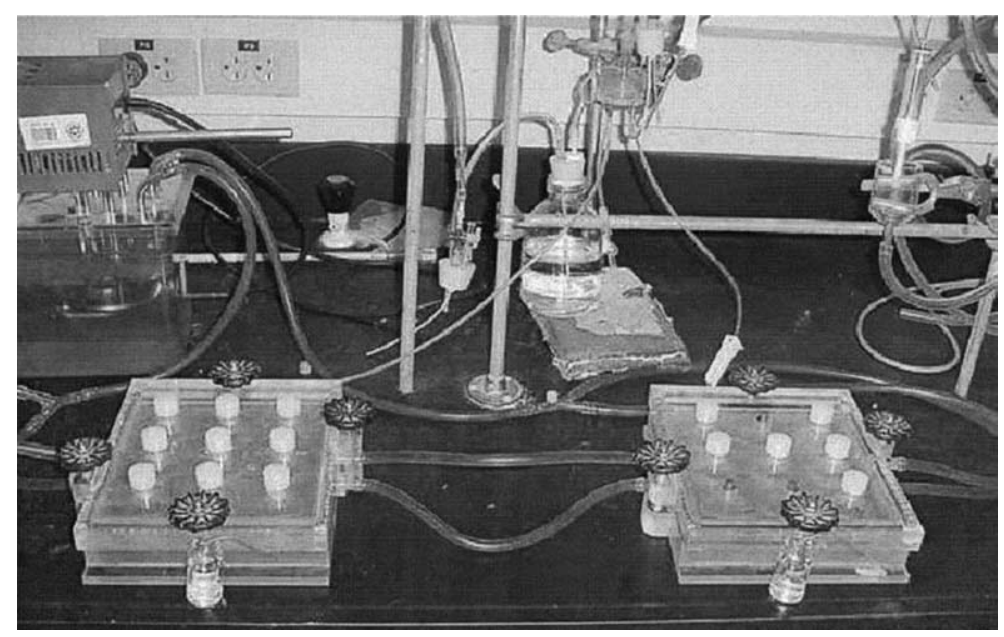

Figure 1. Sealed Plexiglas chamber.

\section{Assessment of Tissue Injury and Viability}

At the end of each experimental protocol, tissue viability was determined by MTT staining. The reduction of MTT to blue formazan is catalyzed by mitochondrial dehydrogenases, and the intensity of MTT staining in myocardial sections is correlated with the viability of the tissue. ${ }^{14}$

MTT was present for the entire period of the reoxygenation. Two atrial sections were incubated in $1 \mathrm{~mL}$ G-PBS containing MTT $(0.5 \mathrm{mg} / \mathrm{mL})$ at $37^{\circ} \mathrm{C}$ for 90 minutes. The sections were then transferred to a small test tube containing $3 \mathrm{~mL}$ saline solution and shaken for 1 minute to remove excess dye. The sections were wiped on gauze cloth and transferred to $1.5-\mathrm{mL}$ plastic test tubes and frozen overnight. Extraction of the formazan dye into $1 \mathrm{~mL}$ dimethylsulfoxide was done with vigorous shaking for 1 hour at $37^{\circ} \mathrm{C}$. The colored supernatant was measured spectrophotometrically at $500 \mathrm{~nm}$. The sections were dried in a $90^{\circ} \mathrm{C}$ oven for 24 hours. Staining results were expressed as optical density/mg dry weight of myocardial tissue. Tissue viability was expressed as the ratio between the I/R optical density and that of the aerobic control specimens normalized to dry weight of myocardial tissue.

\section{Statistical Analysis}

The distribution of all variables was compared between patients who had POAF and those who did not, with the Fisher exact test used for discrete variables and Student $t$ test for continuous variables. Data are expressed as mean \pm SD. Multiple logistic regression analysis for variables with statistical significance in the univariate model was performed to determine the independent predictors for POAF. All statistical analyses were performed with Statistical Package for the Social Sciences version 10.0 (SPSS Inc, Chicago, Ill).

\section{Results}

There were no perioperative deaths in this group of patients. Nineteen patients (38\%) had POAF, developing between postoperative day 1 and postoperative day 5 (median postoperative day 3 ). The mitochondrial function, recorded from right atrial slices that were collected and kept under normal conditions (aerobic control baseline function; Figure 2), revealed no significant differences between atrial slices collected from patients who had POAF and those who did not $(0.62 \pm 0.12$ vs $0.64 \pm 0.08, P>.05)$.

Interestingly, a significant difference was documented between the two groups of patients after the simulated I/R phase. Atrial slices obtained from patients who had POAF exhibited a significantly impaired mitochondrial function and response to simulated $\mathrm{I} / \mathrm{R}$ than did those from patients with no POAF $(0.19 \pm 0.06$ vs $0.26 \pm 0.05, P=.003$; Figure 2). The ratio between the mitochondrial function after simulated I/R and the baseline values (aerobic control) represented an even more profound difference between the groups of patients $(28.8 \pm 9 \%$ vs $39.2 \pm 14 \%, P=.001$; Figure 3). In a univariate model (Table 2), the significant variables related to POAF $(P<.05)$ were as follows: female gender, right coronary artery disease, type of surgery (coronary artery bypass grafting or aortic valve replacement), nonuse of $\beta$-blockers, MTT staining after I/R, and ratio between MTT staining after I/R and baseline performance. In multivariate analysis the only variable significant as predictor for POAF was the ratio between MTT staining after I/R and baseline ( $P=.027$ in multivariate analysis). The classification table (Table 3) based on the logistic regression analysis model demonstrates relatively high predictive accuracy for POAF, with a specificity of $89.7 \%$ and a sensitivity of $85 \%$. There is, however, a need for a much larger group of patients to establish the real predictive value of the MTT staining test.

No correlation was found between MTT staining and ratio (I/R to baseline) and other clinical factors such as age, chronic obstructive pulmonary disease, diabetes, extent of cardiac disease, type of operation, CPB time, or crossclamp time $(P>.05)$. 


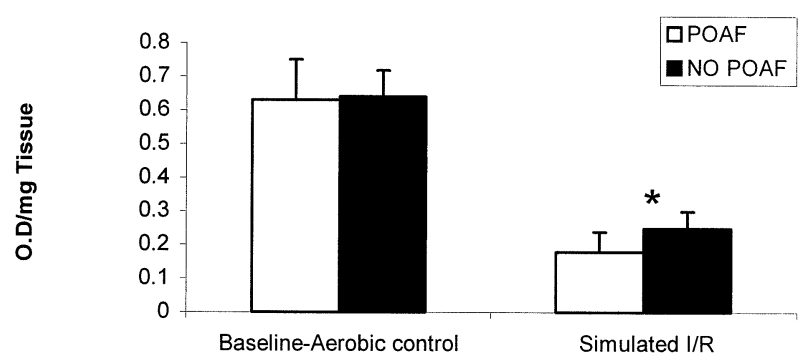

Figure 2. Mitochondrial response to simulated $I / R$ injury. $O D$, Optical density; $I / R$, ischemic reperfusion; $P O A F$, postoperative atrial fibrillation. Asterisk indicates $\boldsymbol{P}=.003$.

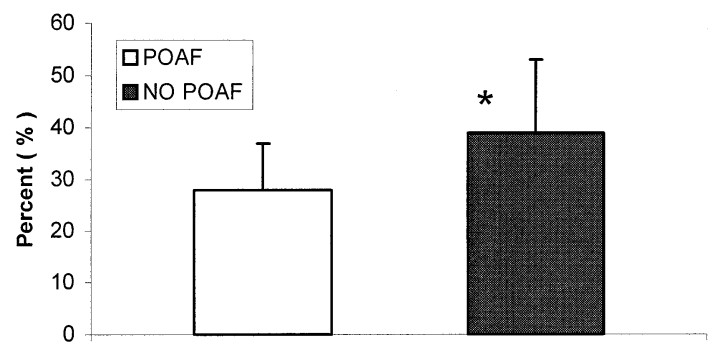

Figure 3. Mitochondrial function ratio between after simulated I/R and baseline aerobic control. I/R, Ischemic reperfusion; POAF, postoperative atrial fibrillation. Asterisk indicates $\boldsymbol{P}=.001$.

\section{Discussion}

Recent reports have shown that POAF constitutes a major clinical problem and may lead to severe sequelae and high costs. Risk factors have been identified and may assist in defining a group of patients who are at a greater risk for this common complication. ${ }^{1-3}$ Until now, however, this progress has not been reflected in any treatment regimen, and all patients in a given institution get similar preoperative and postoperative preventive antiarrhythmic treatment, irrespective of risk of POAF and with minimal success in reducing its incidence. A possible reason for this discrepancy is our lack of comprehension of the molecular and metabolic processes involved.

MTT has the property of acting as hydrogen acceptor to be reduced by dehydrogenases enzymes. MTT reduction of a viable tissue is related also to the presence of coenzymes nicotinamide adenine dinucleotide and nicotinamide adenine dinucleotide phosphate. It is a simple and reliable method correlated with ischemic damage and mitochondrial dysfunction in both atrial and ventricular myocardium. In addition, MTT assay can be used to assess myocardial oxygen consumption and energetic index. ${ }^{15}$ Studies have shown that oxidative stress may lead to compromised mitochondrial redox function as indicated by the MTT assay. ${ }^{13,16,17}$
TABLE 2. Univariate analysis

\begin{tabular}{lc}
\hline Variable & $\boldsymbol{P}$ value \\
\hline Age & .756 \\
Gender & .005 \\
Chronic obstructive pulmonary disease & .335 \\
Smoking & .587 \\
Hypertension & .745 \\
Diabetes mellitus & .506 \\
Hypercholesterolemia & .851 \\
Acute myocardial infarction & .087 \\
Right coronary artery disease & $<.0001$ \\
Incomplete revascularization of both systems & .303 \\
Incomplete revascularization of left coronary system & .403 \\
Incomplete revascularization of right coronary & .303 \\
$\quad$ system & \\
Ejection fraction & .09 \\
Congestive heart failure & .931 \\
Renal failure & .229 \\
Coronary artery bypass grafting & $<.0001$ \\
Aortic valve replacement & .019 \\
Mitral valve repair & .465 \\
Mitral valve replacement & .46 \\
CPB time & .32 \\
Crossclamp time & .16 \\
History of atrial fibrillation & .737 \\
Preoperative use of $\beta$-blockers & .554 \\
Preoperative use of angiotensin-converting enzyme & .729 \\
$\quad$ inhibitors & .77 \\
Preoperative use of oral antiglycemic drugs & .973 \\
Preoperative use of statins & .755 \\
Preoperative use of digoxin & .331 \\
Nonuse of $\beta$-blockers after operation & .05 \\
MTT staining & .015 \\
$\quad$ Control & \\
After l/R & \\
I/R to baseline ratio & \\
\hline &
\end{tabular}

TABLE 3. Classification table for POAF

\begin{tabular}{|c|c|c|c|}
\hline \multirow[b]{3}{*}{ Observed POAF } & \multicolumn{3}{|c|}{$\begin{array}{l}\text { Predicted according to logistic regression } \\
\text { model }\end{array}$} \\
\hline & \multicolumn{2}{|c|}{ POAF } & \multirow{2}{*}{$\begin{array}{c}\text { Percentage } \\
\text { corrected }\end{array}$} \\
\hline & No & Yes & \\
\hline No & 26 & 3 & $89.7 \%$ \\
\hline Yes & 3 & 17 & $85 \%$ \\
\hline
\end{tabular}

The cut value was 0.5 . One patient was dropped from the model because of a missing variable.

This study has pointed out for the first time an association between POAF and mitochondrial dysfunction in response to simulated I/R. It has demonstrated that impaired response of right atrial mitochondria to simulated I/R may predict the development of POAF. Several studies have shown that ischemia or hypoxia followed by reperfusion causes loss of both cytosolic and mitochondrial glutathione, resulting in lack of recovery of contractile activity. ${ }^{18,19}$ The 
finding of an association between deletion of mitochondrial DNA aging and atrial fibrillation has led to the assumption that impaired mitochondrial function in cardiomyocytes may be related to atrial fibrillation and aging. ${ }^{11}$ Such a process leads to bioenergetic deficiency as a result of impaired synthesis in the human atrium. Other studies have shown the association between atrial fibrillation and impaired mitochondrial function in the atrium and alteration in specific adenosine triphosphatase activity, energy metabolism, and upregulation of heart failure markers such as endothelin 1 and atrial natriuretic peptide. ${ }^{11,20}$ Another report has revealed that mitochondrial dysfunction in the cardiomyocytes may cause impairment of cellular energy metabolism and induced apoptosis. ${ }^{21-23}$ It is therefore reasonable to hypothesize that susceptibility to POAF may depend on the preoperative metabolic status of the atria and mitochondrial function and that it might be predictable on the basis of morphologic parameters in preoperative histologic atrial specimens and mitochondrial function.

The incidence of atrial fibrillation after off-pump coronary artery bypass grafting surgery is not lower than that after surgery performed with the use of a heart-lung machine. This finding, coupled with the fact that in this study the model was predicted for patients after surgery for valve disease as well as coronary artery bypass grafting, encourages us to support the assumption that this complication is not directly related to the type of surgery or the operative technique but rather to preoperative atrial pathology that is the result of the disease process in a given patient.

Our study has obvious limitations. Its size is limited, and no mechanism to explain the findings has been suggested. Because of the small size, classic parameters involved with POAF such as age and chronic obstructive pulmonary disease did not reach statistical significance. However, it demonstrates an important phenomenon, namely that POAF is associated with mitochondrial dysfunction in response to simulated I/R injury. It has been well discussed in many articles that mitochondria are involved in the ultrastructural changes appearing in the myocardial cells as consequence of oxygen deprivation. Mitochondrial protection during $\mathrm{I} / \mathrm{R}$ or better mitochondrial function in response to such insult may therefore result in improved myocardial function and may also have antiarrhythmic effect. Studies are now required to dissect the molecular events and changes in gene expression that lead to the increased susceptibility. Such studies may also enhance the identification of preoperative treatments to improve the preoperative metabolic status of the atrial cells and reduce the risk for POAF.

\section{References}

1. Hogue CW, Hyder ML. Atrial fibrillation after cardiac operation: risks, mechanisms, and treatment. Ann Thorac Surg. 2000;69:300-6.
2. Creswell LL, Schussler RB, Rosenbloom M, Cox JL. Hazards of postoperative atrial arrhythmias. Ann Thorac Surg. 1993;56:539-49.

3. Almassi GH, Schowalter T, Nicolosi AC, Aggarwal A, Moritz TE, Henderson WG, et al. Atrial fibrillation after cardiac surgery: a major morbid event? Ann Surg. 1997;226:501-13.

4. Cox JL. A perspective of postoperative atrial fibrillation in cardiac operation. Ann Thorac Surg. 1993;56:405-9.

5. Aranki SF, Shaw DP, Adams DH, Rizzo RJ, Couper GS, VanderVliet $\mathrm{M}$, et al. Predictors of atrial fibrillation after coronary artery surgery: current trends and impact on hospital resources. Circulation. 1996;94: 390-7.

6. Zaman AG, Archbold RA, Helft G, Paul EA, Curzen NP, Mills PG. Atrial fibrillation after coronary artery bypass surgery: a model for preoperative risk stratification. Circulation. 2000;101:1403-8.

7. Groves PH, Hall RJ. Atrial tachyarrhythmias after cardiac surgery. Eur Heart J. 1991;12:458-63.

8. Ad N, Snir E, Vidne BA, Golomb E. Potential preoperative markers for the risk of developing atrial fibrillation after cardiac surgery. Semin Thorac Cardiovasc Surg. 1999;11:308-13.

9. Ad N, Snir E, Vidne BA, Golomb E. Histological atrial myolysis is associated with atrial fibrillation following cardiac surgery. Ann Thorac Surg. 2001;72:688-93.

10. Kitzman DW, Edwards WD. Age-related changes in the anatomy of the normal human heart. J Gerontol. 1990;45:M33-9.

11. Tsubi M, Hisatome I, Moriaski T, Tanaka M, Tomikura Y, Takeda S, et al. Mitochondrial DNA deletion associated with reduction of adenine nucleotides in human atrium and atrial fibrillation. Eur J Clin Invest. 2001;31:489-96.

12. Bernardi P. The permeability transition pore. Control points of cyclosporine A-sensitive mitochondrial channel involved in cell death. Biochim Biophys Acta. 1996;5:1275-83.

13. Ghosh S, Standen NB, Galinanes M. Preconditioning the human myocardium by simulated ischemia: studies on early and delayed protection. Cardiovasc Res. 2000;45:339-50.

14. Vemuri R, Yagev S, Heller M, Pinson A. Studies on oxygen and volume restrictions in cultured cardiac cells. I. A model for ischemia and anoxia with a new approach. In Vitro Cell Dev Biol. 1985;21: 521-5.

15. Ferrera R, Larese A, Berthod F, Guidollet J, Rodreiguez C, Dureau G, et al. Quantitative reduction of MTT by hearts biopsies in vitro is an index of viability. J Mol Cell Cardiol. 1993;25:1091-9.

16. Ghosh S, Standen NB, Galinianes M. Failure to precondition pathological human myocardium. J Am Coll Cardiol. 2001;37:711-8.

17. Liang FQ, Godley BF. Oxidative stress-induced mitochondrial DNA damage in human retinal pigment epithelial cells: a possible mechanism for RPE aging and age-related macular degeneration. Exp Eye Res. 2003;76:397-403.

18. Cavallini L, Valente M, Rigobello MP. The protective action of pyruvate on recovery of ischemic rat heart: comparison with other oxidizable substrates. J Mol Cell Cardiol. 1990;22:143-54.

19. Rigobello MP, Bindoli A. Effect of pyruvate on rat heart thiol status during ischemia and hypoxia followed by reperfusion. Mol Cell Biochem. 1993;122:93-100.

20. Barbey O, Pierre S, Duran MJ, Sennoune S, Levy S, Maixent JM. Specific up-regulation of mitochondrial F0F1-ATPase activity after short episodes of atrial fibrillation in sheep. J Cardiovasc Electrophysiol. 2000;11:432-8.

21. Mela-Riker LM, Bukoski RD. Regulation of mitochondrial activity in cardiac cells. Annu Rev Physiol. 1985;47:645-63.

22. Yoshihiko K, Takashi M, Koichi Y, Nobuyuki M, Katsutoshi G, Iwao Y. Mitochondrial dysfunction of cardiomyocytes causing impairment of cellular energy metabolism induces apoptosis and concomitant increase in cardiac endothelin 1 expression. J Cardiovasc Pharmacol. 2000;36(Suppl 1):S201-4.

23. Maes A, Flameng W, Nuyts J, Borgers M, Shivalker B, Ausma J, et al. Histologic alterations in chronically hypoperfused myocardium. Correlation with PET findings. Circulation. 1994;90:735-45. 